Revue d'histoire de l'enfance « irrégulière »

Le Temps de l'histoire

9 | 2007

Violences et jeunesse

\title{
Regards du corps et archaïsmes. L'ordonnancement des déviances par la rééducation du corps
}

\section{Francis Mendiague}

\section{(2) OpenEdition \\ 12 Journals}

Édition électronique

URL : http://journals.openedition.org/rhei/2483

DOI : $10.4000 /$ rhei.2483

ISBN : 978-2-7535-1648-9

ISSN : $1777-540 \mathrm{X}$

Éditeur

Presses universitaires de Rennes

Édition imprimée

Date de publication : 15 novembre 2007

Pagination : 191-213

ISSN : 1287-2431

Référence électronique

Francis Mendiague, «Regards du corps et archaïsmes. L'ordonnancement des déviances par la rééducation du corps », Revue d'histoire de l'enfance « irrégulière » [En ligne], 9 | 2007, mis en ligne le 01 novembre 2009, consulté le 04 décembre 2020. URL : http://journals.openedition.org/rhei/2483 ; DOI : https://doi.org/10.4000/rhei.2483 


\title{
Regards du corps et archaïsmes. L'ordonnancement des déviances par la rééducation du corps
}

\author{
Francis \\ Mendiague $^{(1)}$
}

" Il assiste, s'il est catholique, à l'office et à l'instruction élémentaire donnés par deux prêtres de Saint Sulpice. Il trouve ensuite des professeurs de gymnastique, de chant et d'arithmétique qui lui donnent quelques heures. Après une collation, il retourne chez son patron. C'est là aussi qu'il revient au temps de chômage ou quand la maladie lui fait quitter son travail. Tels sont les services rendus par la société de patronage en moralisant et en sauvant de l'abîme un grand nombre d'enfants abandonnés. »

Ces propos humanistes de Paul Cère, en 1872,(2) mêlent éducation du corps et moralisation. Derrière la compassion envers les enfants abandonnés, on perçoit une certaine peur sociale. Il convient donc d'appliquer à l'égard de cette " population dangereuse " un processus de mise en ordre. Ce dernier sera bien différent de l'utopie de Cère.

En effet, l'ordonnancement du corps repose en grande partie sur la dénonciation des corps différents. C'est ainsi que l'identification anatomo-biologique est un mode de classification du corps qui a été utilisé comme modalité de normalisation de tous les enfants. Les corps faibles et tordus sont compris comme des signes de dégénérescence inscrits dans la société, elle-même vue comme un corps dégénéré.

Sur cette base, une éducation physique de l'attitude a été institutionnalisée en France dans les années 1950-1960. On la retrouve dans les centres de rééducation physique, dans les travaux de Vaucresson et aussi dans des expériences pédagogiques modernes de l'époque, comme l'expérience de Vanves. Le cour de cette éducation physique est certes de construire de la normalité,

Francis Mendiague / p. 191 à 213 
(3) Michel Chauvière, Enfance inadaptée ; l'héritage de Vichy, Paris, les Éditions ouvrières, 1980, p. 11.

(4) Gérard Noiriel, Les origines républicaines de Vichy, Paris, Hachette, 1999.

(5) Jacques Dupâquier , "La démographie ", ch. 1, in Histoire de la population en Europe, Paris, Fayard, 1999, p. 48-49.

(6) Michel Foucault, « Le pouvoir psychiatrique ", Cours au Collège de France (1973-1974), Paris, Gallimard/Seuil, 2003.

(7) Georges Vigarello, Le corps redressé, Paris, Delage, 1978. mais aussi de tenter d'inventer des procédés permettant de lire le corps normal/différent. La période traitée s'arrête aux années 1960. Michel Chauvière pense que la décennie 1940-1950 dresse les enjeux principaux et durables : " un socle institutionnel enraciné ». ${ }^{(3)}$ Or, le corps et ses déviances s'enracinent de façon singulière dans la société française à partir du XIXème siècle. Historiquement, depuis cette époque, le corps prend place dans ce que Gérard Noiriel appelle " le fantasme d'identification totale »; ${ }^{(4)}$ il est mis en fiche, il est normalisé. La référence prédominante aux caractéristiques physiques reproduit une image du corps du jeune irrégulier construite pour l'essentiel dans les premières années du XXème siècle par des hygiénistes, et antérieurement par l'anthropologie physique de Lombroso. Jacques Dupâquier note que l'idéologie hygiéniste est à la source du darwinisme social et du néomalthusianisme. ${ }^{(5)}$ Il voit ce mouvement se disloquer vers la fin des années 1910. Institutionnellement, il a raison. Mais, dans les trente premières années de ce siècle, le regard sur le corps des vagabonds, des délinquants, est le lieu de prédilection de thèses proches de celles du darwinisme social, dans une société en crise économique, politique et sociale, qui s'effraie des dégâts humains non réparés de la première guerre mondiale et qui cherche des coupables et des moyens de s'en sortir.

Le rapport du corps à une norme contraignante pense tous les individus comme des délinquants potentiels ou des anormaux. Les campagnes des hygiénistes en faveur de l'éducation physique comme moyen de sauver la race (Pinard, Sicard de Plauzoles, Bureau et d'autres) traquent toutes les faiblesses du corps des pauvres et des délinquants, sources de menaces pour la société entière. L'éducation du corps anatomo-biologique concerne l'institution judiciaire, scolaire, médicale et la société dans son ensemble. C'est le grand corps fantasmatique de l'hérédité dont parle Foucault, ${ }^{(6)}$ celui de la peur. Il differe du corps redressé qu'étudie Vigarello, ${ }^{(7)}$ auquel on pense naturellement lorsqu'il s'agit d'étudier les identifications anatomo-physiologiques et ses rapports avec la gymnastique.

Nous montrerons comment l'éducation physique au sens le plus large, dans l'institution scolaire, dans le monde médical, judiciaire ou dans la société ellemême, construit, par strates successives, pendant près de cinquante ans, une relation complexe au corps. Ce corps est historiquement constitué par un rap- 
port à des normes sociales, à des références religieuses, à des droits et des devoirs qui l'inscrivent dans des préoccupations sociales décrites en terme de race, de dégénérescence, de faiblesse et de normalité. Ces concepts construisent un regard sur le corps des délinquants, dans une période couvrant les cinquante premières années du XXème siècle. Ils nourrissent une idéologie de la peur du corps et des différences.

\section{L'éducation du corps et les ennemis de l'intérieur}

L'ordonnancement/dénonciation du corps a une profondeur historique. Lombroso ${ }^{\left({ }^{8}\right)}$ affirme que le corps révèle les caractères des criminels nés : la sensibilité est obtuse, la gaucherie plus fréquente, les réflexes sont irréguliers. Audelà des divergences des uns et des autres, l'idée assez largement partagée au congrès d'anthropologie criminelle de Paris est que la dégradation physique (concept non précisé et qui renvoie aux remarques de Lombroso) révèle la dégradation morale. Manouvrier, qui défend les thèses françaises, conteste que l'anatomie seule puisse caractériser les criminels : "L'homme sain et normal n'est pas l'homme sans défaut et sans tendance au vice ", la catégorie des honnêtes gens fourmille de paresseux, d'imbéciles, d'intrigants, de malhonnêtes, de fourbes, de brutaux et de criminels non qualifiés par la loi ; " avec une force musculaire plus grande, un peu plus d'audace ", l'honnête homme peut devenir un malfaiteur redoutable. Une mauvaise éducation du corps peut donc avoir des effets pernicieux. ${ }^{(9)}$

À l'inverse, une gymnastique bien conçue peut fournir de l'ordre et de l'aide face au chaos menaçant toute la société. La gymnastique propose un traitement efficace dans la thérapie de dégénérés, affirme Tissié en 1894. Elle contribue à réguler l'instabilité mentale. Elle modifie les tendances à l'émotivité morbide, à l'onanisme et elle supprime les fugues. ${ }^{(10)}$ C'est la thèse laïque du corps envisagé comme une barrière protectrice s'efforçant de « parquer la sensualité ». L'éducation du corps antomo-biologique concerne l'institution judiciaire, scolaire, médicale et la société dans son ensemble.

La ligue civique appelle à lutter contre " les ennemis de l'intérieur » qui se voient, selon elle, dans la déchéance physique et sociale. ${ }^{(1)}$ Dans cette représentation sociale, les tares se voient sur les corps et les corps coupables sont principalement ceux des pauvres, chez qui les influences défavorables agissent
(8) Cesare Lombroso, in Actes du deuxième Congrès international d'anthropologie criminelle (Paris, 1889), Lyon, A. Storrk, 1890, p. 26.

(9) Léonce Manouvrier, in Actes du deuxième Congrès international d'anthropologie criminelle, op. cit., p. 32-35.

(10) Philippe Tissié, in Actes du deuxième Congrès national de la Ligue girondine d'éducation physique, Bordeaux, Gounouilhou, 1894, p. 32.

(11) Bulletin $n^{\circ} 11$, 30 janvier 1919, Paris. 
(12) Maurice Landrieux

(Mgr), « Du laïcisme au paganisme. Lettre pastorale ", Documentation catholique, $\mathrm{n}^{\circ} 2,1924$.

(13) Raoul Girardet, Le nationalisme français, Paris, Seuil, coll. Points, 1983, p. 31.

(14) Georges Weill, L'Europe du XIXème siècle et l'idée de nationalité, Paris, Albin Michel, 1938.

(15) Jules Payot (1906), in Laurence Loeffel, La question du fondement de la morale lä̈que sous la IIIème République, I870-I9I4, Paris, PUF, 2000, p. 121. de façon prolongée pendant des générations et contrarient le renouvellement sain de la société. C'est la dimension héréditaire de la délinquance, vue à travers l'intrication de prédispositions organiques et physiologiques et d'un milieu social singulier. Au sein de ce dernier, le physique et le moral, évidemment dégradés sont des sources de péril pour la société. La relation entre le taudis, la tuberculose, l'alcoolisme, les maladies vénériennes et la diminution de la natalité, est évidente selon Mgr Landrieux, évêque de Dijon. ${ }^{(12)}$ Le prélat demande que des mesures soient prises pour protéger la race. À défaut de moyens précis, un modèle corporel est censé répondre au danger qui la menace Il s'agit du modèle masculin du corps protecteur, fort, vertueux, proportionné, résultat de la victoire de la volonté sur les penchants naturels. Il a été construit à la suite de la défaite de 1870 et de la crainte de disparition qui s'en est suivie. ${ }^{(13)}$ Dans certains cercles de la société française, un lien confus est établi entre la nation et l'idée de race, au sens physiologique du terme. ${ }^{(14)}$ Le coût humain terrible de la première guerre mondiale va être à l'origine d'une campagne de repopulation et de moralisation de la société, qui va développer la thèse du rôle du corps sain permettant la nécessaire régénération de la race. La frontière entre hygiène sociale et hygiène raciale est bien mince ; parfois elle disparaît totalement.

Les méthodes de gymnastique qui se développent, fin XVIIème-début XIXème siècles, avec Guts Muth, Pestalozzi et surtout Ling et les gymnastes français du XIXème siècle vont à leur tour donner crédit à la thèse de la valeur totale du corps fort et proportionné. Elles vont construire une gymnastique du corps segmenté, reposant sur le mouvement localisé, exécuté de façon parfaite, qui dresse et redresse le corps. Les républicains laïques comme Ferry et Paul-Bert, la Ligue de l'enseignement, les sociétés de préparation militaire à partir de 1905, lorsque le service militaire devient obligatoire pour tous (loi du 26 mars 1905), les hygiénistes, vont réorganiser cette thèse de la valeur du corps en lui donnant une dimension républicaine et sociale. La laïcité fera de l'hygiène du corps une vertu, une « condition essentielle de l'énergie morale ". ${ }^{(15)}$ Léducation physique fortement moralisatrice prend place dans la société. Le corps-laïque est envisagé comme une barrière protectrice s'efforçant de " parquer la sensualité ». Il est le résultat de la victoire de la volonté sur les penchants naturels. Ce modèle masculin du corps protecteur, fort, vertueux, proportionné, va se fondre alors dans le concept très complexe de la race. 
Le sentiment de peur lié au corps va être amplifié par les épidémies de choléra du XIXème siècle. La tuberculose et la syphilis seront, au début du XXème siècle, deux symboles forts des périls du corps. Leur propagation est souvent associée aux désordres corporels des couches pauvres de la société. Les faibles, les étrangers, les instables, les fugueurs, les délinquants, sont considérés comme faisant partie d'une race inférieure. Certains discours excessifs sur les dangers que feraient courir à l'ensemble de la société les plus faibles, font se rejoindre de nombreuses personnes, dont beaucoup sont des républicains, convaincus d'œuvrer pour le bien de tous. Ainsi, des économistes, des démographes, des médecins, des hommes politiques, des universitaires s'interrogent-ils sur ce que Nisot nomme « [...] l'accroissement progressif du nombre des tarés de toute espèce, [...] la diminution de la natalité dans les classes supérieures, l'augmentation continue des couches inférieures de la population, susceptible d'engendrer un renouvellement de la société par le bas ". ${ }^{(16)}$

Dans cette représentation sociale, les tares se voient sur les corps et les corps coupables sont principalement ceux des pauvres, car ils contrarient le renouvellement sain de la société. Ce corps qui lie les individus dans la succession des générations, ce que Monod ${ }^{(17)}$ appelle la téléonomie, établit un lien vicié et dangereux. Dans cette perspective, et dans une vision anthropométrique, il devient l'image de l'altération transmise et transmissible. Parfois, dans une vision plus globale, le corps du délinquant est compris comme le résultat du dysfonctionnement de l'ensemble de la société.

Mais, dans tous les cas, il est perçu comme présentant un risque pour la qualité de la race, comme un facteur de dégénérescence. Paul-Boncour, Pinard, Richet et Heuyer, dans les trente premières années du XXème siècle, sont les plus fervents défenseurs de cette thèse. Cette dernière perdurera, en partie, dans les années 1950. Comprendre ce corps, savoir lire ses défauts et éventuellement y remédier par une éducation physique appropriée, en particulier dans les centres de rééducation physique, prend une dimension d'urgence sociale.

\section{L'importance pédagogique de l'examen du corps. Le passage du faible au dégénéré}

C'est à Binet, au début du XXème siècle, que nous devons une des premières formes modernes d'observation du corps des enfants instables, les
(16) Marie-Thérèse

Nisot, La question eugénique dans divers pays, Bruxelles, G. Van Campenhout, 1927 , p. 9.

(17) Jacques Monod, Le hasard et la nécessité, essai sur la philosophie naturelle de la biologie moderne, Paris, Seuil, 1970. Par ce concept, Monod a voulu se démarquer d'une approche mystique de la téléologie (l'étude des fins de l'homme). Pour Monod, l'évolution est l'accomplissement d'un projet téléonomique essentiel, la transmission d'une génération à l'autre. C'est le projet caractéristique de l'espèce. Cela concerne les adaptations biologiques. 
(18) Alfred Binet,

Les idées modernes

sur l'éducation, Paris,

Flammarion, 1918, p. 60

(19) Ibid., p. 58.

(20) Ibid., p. 57.

(21) Ibid

(22) Ibid., p. 61.

(23) Ibid retardés scolaires. Il propose de combiner les mesures anthropométriques et un regard attentif sur le corps de ces enfants, afin de pouvoir bien les distinguer et ainsi adapter un traitement destiné à leur venir en aide, ou tout au moins "éveiller l'attention du pédagogue ». ${ }^{(18)}$ C'est ce qu'il appelle les idées modernes sur l'éducation. L'idée de combiner plusieurs types d'approches, dictée par l'état des connaissances anthropométriques de l'époque, ne permettait pas de conclure à l'existence de liens avec l'intelligence de l'écolier. Mais "sans doute des corrélations existent ", pense-t-il. ${ }^{(19)}$ Après avoir examiné 600 enfants du primaire, il conclut « que les avancés-intellectuels, sont plus nombreux parmi les avancés-physiques que parmi les retardés-physiques ". ${ }^{(20)}$ Cette règle ne se vérifie pas sur un petit groupe (il y a $21 \%$ de chétifs parmi les avancés-intellectuels), mais plutôt dans les grands nombres, remarque-t-il.

Binet s'inscrit néanmoins dans le courant hygiéniste qui associe le corps des pauvres, des faibles et des délinquants à la mise en danger de la race. Il entend construire sur les enfants un savoir total. Il diffuse largement ses idées dans le système primaire, par les réunions qu'il anime, par son enseignement et par les revues auxquelles il collabore. Binet est, en France, le créateur de la psychologie et de l'orientation scolaires. Il a une position intellectuelle forte. Il est à l'origine de la constitution d'un savoir spécifique sur les élèves en difficulté scolaire. Ce savoir concerne l'intelligence, mais aussi le corps. À travers le développement physique, mesuré en partie par les données anthropométriques, "l'avenir de notre race et l'organisation de notre société » ${ }^{(21)}$ sont en jeu, dit-il. Il place ainsi l'étude du corps dans une perspective démographique et sociale, domaine bien plus large que les 600 élèves de son enquête, dans laquelle il postule des corrélations visibles entre le physique et l'intellectuel.

Le corps des élèves est un sujet d'inquiétude. Il faut apprendre à le regarder. Le regard est, pour Binet, un instrument de mesure sûr. Toutefois, il recommande de ne pas pratiquer les examens corporels avec un état d'esprit trop optimiste, qui fausserait l'acuité du regard. Lors de l'examen du corps, le regard doit se porter sur " l'attitude du corps, la coloration de la peau, du visage, la forme et l'expression des traits ». ${ }^{(22)}$ Le corps des enfants instables, des retardés scolaires, se distingue clairement de celui des autres enfants, car il dégage " une impression indéfinissable de misère physiologique $»^{(23)}$ 
Les enfants de pauvres, qui constituent la majorité des enfants à problèmes, sont reconnaissables à leur corps moins développé, " chétif, maigre [...] au système nerveux mal équilibré ". Si le regard est attentif, on peut y voir " un tube digestif qui digère mal, un estomac dilaté, un sang qui n'est pas assez riche ».(24) L'examinateur verrait donc l'intérieur du corps. Binet octroie ainsi aux éducateurs un pouvoir redoutable sur le corps des enfants difficiles. En même temps, il contribue à déposséder ces enfants de leur propre corps, qui n'est défini qu'en termes négatifs au regard de la personne, de la race et de l'ordre social.

Ce corps des enfants à problèmes, qui résulte d'une double misère, physiologique et sociale, produit des déclassés, des mécontents, des révolutionnaires. ${ }^{(25)}$ Ces résultats sont, dit-il, identiques à ceux obtenus dans d'autres pays, par de nombreux chercheurs.

Les chétifs, les malingres, les enfants dont le développement du corps est retardé sont issus de parents " de condition pauvre et même misérable ». ${ }^{(26)}$ Dans ce type de raisonnement, le corps faible est celui du pauvre, et il doit changer car il est dangereux pour la société. De ce point de vue, quelle que soit la pensée de l'auteur, et Binet est sincèrement attaché à l'aide aux enfants en difficulté, ce lien qu'il établit entre le corporel et le danger pour la race produit, in fine, une pensée intolérante et discriminatoire. Parmi les auteurs ayant abouti aux mêmes conclusions que lui, Binet cite Niceforo. ${ }^{(27)}$ Ce dernier a étudié, en 1905, « la classe pauvre » en France. Il est un des auteurs favoris de l'anthroposociologue Vacher de Lapouge qui le cite abondamment. Niceforo définit, lui aussi, les enfants pauvres comme faibles de taille, de poids, de périmètre thoracique, de circonférence de tête, de hauteur de front. ${ }^{(28)}$ Il fait de cette faiblesse une infériorité, et de cette dernière, une dégénérescence. Il note que les classes pauvres sont fécondes en dégénérés.

Liant le tout, il en conclut que leur corps faible est la cause principale de leur état social : "L'état misérable est et sera toujours l'effet de leur infériorité physique et mentale. » ${ }^{(29)}$ Appliquant son modèle à l'exode rural, il met en garde contre " les débiles de corps et d'esprit, les paresseux, les alcooliques, les demi-infirmes [...] ce flot impur de dégénérés [qui] arrive en ville ».(30) Le corps des pauvres est devenu celui des dégénérés, en qui Vacher de Lapouge voit « des sauvages primitifs à mentalité trop rudimentaire ». Eugéniste, mal-
(24) Ibid., p. 45.

(25) Ibid., p. 52.

(26) Ibid., p. 61.

(27) Ibid.

(28) Alfredo Niceforo, in Georges Vacher de Lapouge, Race et milieu social : essais d'anthroposociologie, Paris, Marcel Rivière, 1904, p. 61.

(29) Alfred Binet, op. cit., p. 223

(30) Ibid., p. 225. 
(31) Ibid., p. 230.

(32) Alexis Carrel, L'homme cet inconnu, Paris, 1935, rééd. Plon, 1945 , p. 363.

(33) Albert Dussenty, Le vagabondage des mineurs, thèse, université de Toulouse, faculté de droit, 1938.

(34) Joseph-Marie Timon-David (abbé), Traité de la confession des enfants et des jeunes gens, (1ère éd. 1865), t. 2, Paris, Mignard, rééd. 1924, , p. 57.

(35) Ibid., p. 245.

(36) Ibid., p. 242. thusien, il fait de ces corps chétifs et malingres, faibles, le danger inacceptable pour la régénération de la race. Il sont " les descendants non éliminés des inaptes à la vie civilisée ", ${ }^{(31)}$ qui contrarient le renouvellement satisfaisant de l'espèce humaine, dont ils sont exclus. La régénération de la race ne peut passer par eux, lorsqu'on a comme projet la sélection de l'espèce par "l'aristocratie héréditaire » qu'appelle de ses vœux Carrel. ${ }^{(32)}$

La dégénérescence du corps, visible par des malformations diverses, devient la caractéristique des délinquants, de tous ceux qui constituent, selon VanEtten en 1937, l'adolescence coupable. Pour Dussenty, dans sa thèse de droit, " le nombre des dégénérés est très grand "; la dégradation du corps, associée " aux tares nerveuses ", est la cause essentielle du vagabondage des mineurs. ${ }^{(33)}$ Il appelle à la création de centres de tri et d'observation de la jeunesse vagabonde, en difficulté sociale et personnelle.

\section{Le corps délinquant et ses archaïsmes : le mou, le pervers et l'instable}

Les approches de l'observation du corps du délinquant par Paul-Boncour, médecin travaillant sur les enfants en danger, par Timon-David, prêtre s'occupant de la jeunesse ouvrière à Marseille, et par Heuyer, grand spécialiste de la psychiatrie infantile, different peu entre elles. Elles reposent sur la thèse d'un corps en danger, parce que sa constitution, faible ou molle, ne le protège plus.

Cette absence de protection ouvre le corps sur la perversité. Les actes coupables laissent des traces physiques que le confesseur doit savoir déchiffrer, dit l'abbé Timon-David. La physiognomonie de Lavater lui semble plus appropriée que la phrénologie de Gall. Néanmoins, elle a tendance à trop focaliser le regard sur le visage. C'est l'ensemble du corps qu'il faut observer, cherchant, par ce biais, à découvrir la vérité de l'âme, car « toutes les âmes sont semblables ; mais enfermées dans des corps dont elles subissent les impressions, elles sont molles avec les tempéraments lymphatiques ». ${ }^{(34)}$ Pour Timon-David, le tempérament lie l'âme et le corps. Ce dernier peut être une protection contre le passage à l'acte. Il peut aussi, au contraire, le faciliter et emporter le sujet dans la voie sans issue de la perdition : "ces actes premiers qui peuvent conduire au dernier $»{ }^{(35)}$ N'est-ce pas la faiblesse physique qui facilite « les actes mauvais ", s'interroge-t-il ? ${ }^{36)}$ 
Pour lui, chez les faibles, la barrière protectrice du corps ne joue plus son rôle, et la sexualité emporte les jeunes : "Ils se touchent sans retenue, prennent des poses lascives, se procurent des mouvements déréglés. » ${ }^{(37)}$ Les critères observables sont les mouvements et les attitudes du corps, qui sont les signes visibles du dérèglement intérieur.

Ce schème corporel va être repris sous diverses formes quasiment jusqu'aux années 1960. L'incoordination et l'attitude du corps vont être compris comme les signes évidents de perversion, de dérèglement sexuel, luimême analysé comme signe de déviance. Les jeunes ainsi marqués ne peuvent pratiquer d'activités physiques « comme nager, monter à cheval, sans éprouver quelquefois des effets mauvais ».(38) La démarche molle et lente des flegmatiques, leur regard méfiant, permettent de les reconnaître. Ce sont eux « les faiseurs de complots dans les collèges, les boute-en-train des révoltes ». ${ }^{(39)}$ Leurs âmes sont incurables, c'est pourquoi " ils vont facilement jusqu'au crime ».(40) La rupture de la barrière du corps, les chairs molles, la raideur qui bloque les mouvements sont des signes prédictifs de délinquance.

La vision chrétienne, empirique, du corps coupable de Timon-David repose sur deux axes, intimement liés, chacun pouvant être observé séparément et décomposé en de multiples sous parties : le corps instable non maîtrisé et une sexualité onaniste et/ou homosexuelle.

Par ailleurs, des facteurs sociaux peuvent faciliter les dérèglements de la chair, les extrêmes désordres qui ne s'arrêtent " qu'après l'entier épuisement du mal ». ${ }^{(41)}$ De là découle l'importance du milieu social sain, respectant les principes religieux.

La perversion qui, avec la dégénérescence, singularise " les petits dévoyés [...] l'enfance tarée » est une catégorie d'analyse particulièrement floue, qui entre dans cette élaboration secondaire dont parle Fédida. ${ }^{(42)}$ Elle renvoie au monde de la peur du corps comme univers incontrôlable secrétant ses propres codes, essaimant dans les autres corps. C'est l'univers fantasmatique par excellence. Pourtant, la perversion est un concept souvent utilisé dans de nombreuses études sur la jeunesse irrégulière.

Les fugueurs et les vagabonds sont " des pervers instinctifs ", affirme Dussenty dans sa thèse, qu'il inscrit dans la modernité que représente la loi de 1935 en substituant aux mesures antérieures d'enfermement des mesures
(37) Ibid., p. 244.

(38) Ibid., p. 247.

(39) Ibid., p. 74.

(40) Ibid., p. 73.

(41) Ibid., p. 253.

(42) Pierre Fédida,

" L'anatomie dans

la psychanalyse ", Nouvelle

Revue de psychanalyse,

$\mathrm{n}^{\circ} 3$, "Lieux du corps ",

1971, p. 117. 
(43) Albert Dussenty, op. cit., p. 79.

(44) Ibid., p. 21.

(45) Ibid.

(46) Ibid.

(47) Ibid., p. 19

(48) Georges PaulBoncour, in L'enfance en danger moral, Comité national d'études sociales et politiques, Paris, 1928, séance du lundi 21 mai 1928, p. 34. Cote BDIC Nanterre O 53250.

(49) Ibid., p. 37.

(50) Henry Van-Etten, "Ce qu'il faut savoir sur le problème de l'enfance coupable ", Pour l'enfance "coupable", 1937, p. 34.

Lauteur fait le point sur cette question en s'appuyant abondamment sur les travaux de PaulBoncour. Etten voit « dans les malformations de la tête, du tronc et des membres des marques de dégénérescence d'origine toxique ou infec- d'assistance et d'éducation pour les vagabonds. Le vagabondage représente désormais « un état pernicieux pour le mineur et susceptible de devenir dangereux pour la société ».(43) L'état pernicieux est synonyme de perversion. Il suscite les mêmes images archaïques. Les vagabonds sont reconnaissables à leur corps : " des squelettes ambulants ", selon Dussenty. ${ }^{\left({ }^{(4)}\right)}$ Leur corps décharné est marqué par l'oisiveté " dissolvante " ${ }^{(45)}$ de leur vie dans les bas quartiers, décrits comme "des villages de boue [...] des termitières ". ${ }^{(46)}$ Ces images sombres renvoient le corps dans le monde souterrain des ombres mi-humaines, quasi irréelles. De ce monde émergent les corps fantasmatiques et dangereux des vagabonds, des pervers, et "l'énorme catégorie d'intermédiaires, de demifous, de quarts ou de trois-quarts fous ». ${ }^{(47)}$

Cette immensité de corps dégénérés menace toute la société, dans son présent comme dans son futur. La conjonction, dans l'analyse de la déviance sociale, des facteurs corporels et sociaux est revendiquée comme une singularité française et comme un renouvellement de l'approche intellectuelle de cette question sociale. La rupture théorique est amorcée dès la fin du XIXème siècle, avec les thèses alors dominantes de Lombroso sur les caractéristiques corporelles prédominantes pour caractériser le criminel né.

Paul-Boncour affirme que la « règle de criminogénèse combine deux facteurs à dose inégale : un facteur mésologique, qui est déclenchant, et un facteur anthropologique ». ${ }^{(48)}$ Le milieu peut être traité par la médecine hygiénique et par l'éducation, en plaçant " ces débiles " dans un bon environnement. Cette approche ne change pas le regard sur le corps des jeunes en difficulté. Les observations que fait Paul-Boncour au laboratoire médico-psychologique de la prison pour jeunes de la Petite Roquette décrivent des arriérés manquant de volonté, des nonchalants, des incrédules et des ironiques.

Le groupe dominant est toutefois celui des instables constitutionnels, qui s'excitent et dont " la viciation s'accentue " ${ }^{(49)}$ s'ils ne sont pas pris en charge par une éducation physique, hygiénique. En réalité, le renouvellement théorique est bien mince. Le corps du jeune irrégulier est celui d'un pervers, dominé par ses instincts, donc faible nerveusement, manquant de volonté, ce qui est visible par le corps sans tenue et l'attitude d'abandon. La délinquance est un phénomène biologico-social, selon Paul-Boncour. ${ }^{(50)}$ Malgré l'influence néfaste de certains milieux immoraux, il est possible, selon lui, de transformer le corps 
des jeunes par une activité physique appropriée qui fortifiera le corps, perfectionnera les fonctions vitales et disciplinera les centres nerveux. Il faut activer le corps, parce que, comme il a pu le constater, «l'inertie et la station assise prolongée sont génératrices de vices innommables $» .{ }^{(51)}$

La relative prise de distance de Paul-Boncour, de Dussenty ou d'Etten avec les seules références anthropomorphiques se comprend par la valeur qu'ils attribuent à l'éducation, notamment à l'éducation physique. Paul-Boncour pense que, sur les 250.000 anormaux, « 45.000 sont adaptables complètement à la vie en société ». ${ }^{(52)}$ Il croit au pouvoir de l'éducation. C'est une vision républicaine des délinquants et des marginaux, considérés comme éducables et récupérables. Mais c'est aussi une vision du corps travaillée par les thèses hygiénistes, qui, elles, insistent sur les idées de races, de tares et de corps dangereux propagées dans les années 1913-1930 par la société française d'eugénie. Paul-Boncour y a prononcé des conférences dans la période 1922-1926. Heuyer, qui a travaillé avec lui à la Petite Roquette, a aussi participé aux travaux de la société d'eugénie. Lui non plus ne croit pas à la thèse du criminel né. Il demande de distinguer le crime, le vice et la folie. La délinquance touche les enfants normaux, les pervertis, comme les anormaux, dit-il. Mais il y a peu de statistiques, et il demande l'examen systématique des délinquants afin de les classer. La catégorie prédominante est celle des pervers.

L'étiologie que construit Heuyer, ${ }^{(53)}$ avec certaines précautions et souvent avec mesure, differe peu des thèses normatives dominantes. Il constate le nombre considérable de causes psychiatriques à l'entrée dans la délinquance, l'importance du facteur héréditaire et la dimension constitutionnelle des perversions. Les pervers se reconnaissent donc à "l'irréductibilité constante des tendances, l'intimidabilité et le récidivisme inévitable ». ${ }^{(54)}$

Le corps du pervers est impressionnable, soumis aux autres; il ne peut se maîtriser, car il récidive; il n'est donc pas sous l'emprise de la volonté. Enfin, son irréductibilité fait qu'on ne peut en venir à bout. Avec un langage médico-psychologique, le tableau du corps du pervers fonctionne toujours sur la même vision fantasmatique du corps délinquant, un mou emporté par sa sexualité, ce qu'Heuyer traduit par « les manifestations sexuelles, érotisme ou inversion ». ${ }^{(5)}$ On est dans le monde des âmes incurables de Timon-David.

Cette dégradation sexuelle est aussi un fait social : "J'ai été frappé de voir combien il y a à Paris d'enfants pédérastes passifs ", dira Heuyer. ${ }^{(56)}$ Le déter- tieuse ", p. 12. De nombreux délinquants sont, selon lui, « tarés physiquement» (p. 31).

(51) Georges PaulBoncour, op. cit., p. 31.

(52) Ibid., p. 37.

(53) Georges Heuyer, in L'enfance en danger moral, op. cit., p. 39.

(54) Ibid., p. 40.

(55) Ibid., p. 55.

(56) Ibid. 
(57) Maurice

Landrieux, op. cit.

(58) Maurice Didier (dr),

Modèle-toi, Alger, Institut naturiste d'Alger, 1918.

(59) Léon Bourgeois,

(1851-1925), radical plusieurs fois ministre, notamment de l'instruction primaire de 1890 à 1892 , créateur du solidarisme, et Ferdinand Buisson (1841-1932), lui aussi radical, fondateur de la Ligue des droits de l'homme, directeur de l'enseignement primaire pendant 17 ans, sont les représentants de la république laïque.
(60) Pour la vie. Revue de repopulation et d'hygiène morale et sociale.

\section{(61) Cette ligue}

demande des mesures d'assainissement physique et moral de la jeunesse, entre autres par la pratique de la culture physique, qui devient une pratique préventive et un combat contre l'immoralité sous toutes ses formes. La rue est énoncée comme un monde où pullulent les « tares diverses qui désagrégent la société ». Actes du Tème Congrés national d'assistance publique et privée, NancyParis-Strasbourg, BergerLevrault, 1927, p. 88. minisme social renforce le déterminisme corporel des délinquants. La société, celle de la grande ville, est perverse. La société est décrite comme un grand corps fantasmé, corrupteur et destructeur. La dégénérescence est dans la société. Cette dernière est, par ailleurs, décrite comme un univers d'irréligion, qui ne pense qu'à la jouissance : « Jouir! Orgueil, cupidité, volupté! La chair qui a repris le dessus! Les sens qui dominent ! L'instinct débridé qui gouverne, impérieux, égoïste, brutal ; qui ne connaît plus de mesure. " Ces propos tenus par l'évêque de Dijon, dans sa lettre pastorale ${ }^{(57)}$ par laquelle il dénonce le nouveau paganisme qui, selon lui, aboutit au "suicide de la race ", assimilent la société à un corps pervers. Bien d'autres tiennent des propos similaires. Didier, médecin gymnaste, parle de « la foule des demi-malades qui côtoient les frontières de la pathologie ». ${ }^{(58)}$ La revue Pour la vie, fondée à Paris en 1914 par Bureau, sociologue, moraliste, catholique, lance, en 1919, une campagne pour la remoralisation de la France. Il s'agit de sauver la jeunesse délinquante, pervertie par les rues licencieuses. Des militants catholiques et des laïques, comme Bourgeois et Buisson, ${ }^{(59)}$ participent à cette campagne. Tous militent contre la dépravation de la société. Déjà en 1917, le numéro 25 de cette revue dénonçait les rues devenues des écoles de débauche et de vices, " la laideur morale des grands boulevards [...] la basse pègre $[\ldots]$ les pochards espagnols [...] les annamites ${ }^{(60)}$

La société est une "armée de malades atteints de fléaux sociaux ", dira le docteur Marius Sarraz-Bournet lors du congrès de la Ligue nancéenne pour la protection morale de la jeunesse et la répression de la licence des rues, tenu à Nancy en 1927.(61) Ces propos rejoignent les thèses de Vacher de Lapouge, assimilant les villes " au réceptacle de tous les déchets sociaux », un monde 
de dégénérescence, dans lequel les pauvres constituent le danger ultime, "l'humanité en régression ». ${ }^{(62)}$ L'ultime signe de dégradation de la ville dégénérée, marquant les corps, se verrait, selon la vision hallucinante de Vacher de Lapouge, dans le surprenant nombre de cheveux foncés. Il appelle cela « la puissance nigrifiante du milieu urbain $»{ }^{(63)}$ En fait, il reprend le cliché de la couleur de la peau comme signe de déviance. Lombroso y voit une marque distinctive du criminel occasionnel, avec des réflexes irréguliers et une gaucherie fréquente. ${ }^{(64)}$ On retrouve, de façon récurrente, la même observation sur la peau foncée, sur le teint basané ou coloré que dans les passeports des forçats condamnés-libérés. ${ }^{(65)}$

Les descriptions de la société rejoignent celles de Richet, lorsqu'il dénonce la " race déplorablement chétive, dégradée ». ${ }^{(66)}$ Ce dernier, médecin célèbre, futur président de l'Académie nationale de médecine, futur président de la Société française d'eugénie, rappelle avec Pinard, le créateur de la puériculture, avec Sicard de Plauzolle, vénérologue réputé, et bien d'autres, l'importance sociale de la sélection humaine pour créer, par l'hérédité, une race forte. Pour cela, dit-il, lorsqu'il faudra classer les générateurs, " on aura exclu rigoureusement les débiles, les infirmes, les malades ». ${ }^{(67)}$ La sélection se fera sur des critères physiques, ce qu’il appelle la santé physique, définie par l'intégrité et l'intégralité du corps. ${ }^{(68)}$ Les enfants pauvres, les fugueurs et les délinquants, dont le corps est marqué par les tares physiques et nerveuses, contrarient le renouvellement sain de la race et son perfectionnement. Ils ne peuvent pas faire partie des générateurs.

Les jeunes en marge sont pris dans l'idéologie de la dénonciation. Ils sont les victimes expiatoires d'une élite sociale, celle de la IIIème République, travaillée par la peur et la décadence et qui craint l'émergence de changements sociaux radicaux. C'est dans cette perspective que des personnalités venues d'horizons politiques et culturels bien différents font cause commune. L'amélioration de la natalité, de la population, la lutte contre la mortalité, le perfectionnement de l'être humain par l'exercice physique, sont compris comme des éléments essentiels d'une nécessaire politique publique sociale et éducative. L'hygiénisme, qui rassemble au-delà des clivages politiques ou religieux, "devient une dimension essentielle de la politique sociale républicaine ", note très justement Gérard Noiriel. ${ }^{(69)}$ Par ce biais, ces experts, dont nous avons étudié quelques thèses, participent au pouvoir d'État. ${ }^{(70)}$
(62) Georges Vacher de Lapouge, 1904, op. cit., p. 225.

(63) Ibid., p. 286.

(64) Cesare Lombroso, op. cit., p. 26.

(65) Archives départementales d'Eure-et-Loir, 4MP 518.

(66) Charles Richet, La sélection humaine, Paris, Alcan, 1919, p. 25.

(67) Ibid., p. 25.

(68) Ibid., p. 161.

(69) Gérard Noiriel, op. cit., p. 248.

(70) Ibid. 
(71) Michel Chauvière, op. cit., p. 10 .

(72) Philippe Encausse, Influence des activités physiques et sportives sur le développement intellectuel et physique en milieu scolaire, Paris, Imprimerie nationale, 1957.

\section{Le classement morphologique : corps et comportement, un nouveau savoir dans les années 1950}

Officiellement, le regard médical, scientifique et pédagogique sur le corps change aux abords des années 1950. Pourtant, il est toujours moralisateur. C'est l'héritage de la période de Vichy. En effet, les ordonnances de 1945 reprennent une loi non appliquée de 1942. Michel Chauvière rappelle que " c'est bien le régime de Vichy qui porte et imprègne [...] ce que la Libération se contentera de reconduire et d'étendre ". ${ }^{(71)}$ Le regard devient aussi rationnel. Il cherche la précision et a pour ambition de guider l'action pédagogique et rééducative.

Le droit de regard sur le corps est considéré comme une obligation pédagogique et sociale qui lie les médecins et les enseignants d'éducation physique. Il a pour objet de classer les enfants en fonction de leur morphologie. Il sert à organiser une pédagogie de l'éducation physique adaptée à chaque cas. Sa référence, c'est la biométrie. Elle est au cœur de l'expérience du mitemps pédagogique de Vanves, ${ }^{(72)}$ dirigée par des médecins, et à laquelle Encausse va donner une dimension pédagogique nationale. Médecin, sportif, inspecteur, chef des services médicaux, diplômé d'hygiène scolaire et d'éducation physique, ce dernier est nommé sous-chef de bureau à la jeunesse en 1941. C'est en tant qu'inspecteur général pour la rééducation physique qu’il organise les centres de rééducation physique et qu'il chapeaute l'expérience de Vanves, initiée en 1951, dont il tire les enseignements en 1957. Trois points soulignent, selon lui, le succès de l'expérience de Vanves : la honte de paraître sale à la piscine, la disparition de l'onanisme, ce qu'il appelle « la stabilisation physiologique sexuelle » (p. 23), et la transformation des chétifs malingres, qui n'ont plus de vulgarité d'aspect. Le modèle archaïque du corps délinquant est inclus dans le modèle scolaire.

Les résultats de Vanves dépendent en grande partie des analyses anatomophysiologiques réalisées en début et en fin d'expérience. Cette expérience reprend celle effectuée dans les années 1930 à Lyon, par Latarjet, lui aussi médecin. Elle reposait sur l'idée de la récupération physique du corps des pauvres, affectés, selon lui, de nombreuses tares et posant un problème complexe à la société. À Vanves, on veut démontrer que le développement du corps d'enfants en retard scolaire peut leur permettre de suivre avec succès une scolarité normale. L'enfant difficile devient un inadapté, affecté de perturbations, 
un irrégulier. ${ }^{(73)}$ Son statut change. Son corps n'est plus, en principe, une marque infamante, un espace de perversions quasi indélébiles. Désormais, par une éducation physique globale, on peut toucher des mécanismes perturbés de l'évolution et rééduquer le sujet. Cette problématique fait l'objet d'un colloque de l'UNAR (Union nationale des associations régionales pour la sauvegarde de l'enfance et de l'adolescence), à Dijon, en 1952.

L'éducation physique devient, selon Sinoir, " un poste d'observation ", (74) qui ne doit pas céder "à la facilité de l'étiquetage ». ${ }^{(75)}$ Ce dernier est psychologue attaché à la direction de l'Éducation surveillée. Il fait partie de la commission mise en place à Vaucresson afin d'affiner l'observation en éducation physique, dont l'expérience de Vanves a démontré l'utilité, mais aussi les limites. Il faut élargir les critères d'observation et dépasser le seul univers antomo-physiologique. C'est la personnalité qu'il faut tenter de saisir, car elle est souvent perturbée dans son affectivité « sensible et délicate ». ${ }^{(76)}$ C'est pourquoi, il convient de distinguer les inadaptés organiques et ceux du comportement, les dispositions à la délinquance et l'actualisation de la délinquance. ${ }^{(77)} \mathrm{Le}$ corps du jeune irrégulier ne doit pas être regardé comme celui du mou-pervers menaçant la race. Le guide d'observation que propose Sinoir ${ }^{(78)}$ invite à chercher les manifestations de bonheur, de joie, de ténacité, de goût pour les jeux. La philosophie de l'observation repose sur l'attachement aux principes d'une éducation reposant sur la confiance et sur la compréhension des difficultés vécues par le jeune inadapté.

Comment procède-t-on ? Après un examen précis du corps (taille, poids, capacité vitale, périmètre thoracique, pouls au repos et en activité, envergure, tension au repos et après effort, diamètre abdominal, coefficient abdominal, diamètre thoracique, réflexes, signes pathologiques), on note des remarques générales, comme la caractéristique dominante (impulsivité, la vocation et la culture : amour des bêtes, désir de vie au grand air). Enfin, on procède à une passation de tests de mesure de l'âge mental. ${ }^{(79)}$ On y inclut des informations sur le sommeil et l'appétit.

Ce tableau est complété par des notes écrites sur le comportement pendant les activités physiques. Elles s'efforcent de fournir des informations plus qualitatives, mais aussi plus ambiguës. La résistance du corps est référée aux réactions de la peau au chaud et au froid. La frilosité est considérée comme néga-
(73) Jean-Louis Faure,

"Rôle du maître

d'éducation physique ",

Sauvegarde de l'enfance, $\mathrm{n}^{\circ} 5 / 6$, Paris, UNAR, 1953 , p. 541.

(74) Guy Sinoir, « Les activités physiques "postes d'observation" ", Sauvegarde de l'enfance, $\mathrm{n}^{\circ}$ 5/6, op. cit., p. 535.

(75) Ibid., p. 540.

(76) Ibid., p. 536.

(77) Ibid., p. 537.

(78) Ibid., p. 493-495.

(79) «Monographies ", Sauvegarde de l'enfance, $\mathrm{n}^{\circ}$ 5/6, op. cit., fiche 874 , p. $510-511$. 
(80) Ibid., fiche 880, p. 517.

(81) Ibid., fiche 874 , p. $510-511$.

(82) André de Sambucy, introduction à Ferdinand Belligon, Contribution à la rééducation physique. Un procédé de fichage morphologique, Vénissieux, Julien, 1954, p. 14-16.

(83) Ferdinand Belligon, op. cit., p. 61.

(84) Ibid., p. 33.

(85) Ferdinand Belligon, « Psychisme et tonus ", Sauvegarde de l'enfance, $\mathrm{n}^{\circ}$ 5/6, op. cit., p. 548.

(86) Ibid. tive. Georges, 15 ans, faible au froid, qui recherche volontiers la chaleur, se met en tenue nonchalamment, montre un tempérament lymphatique, une grande frilosité. Il pâlit lors d'un effort soutenu. ${ }^{\left({ }^{(0)}\right.}$ On est dans le stéréotype du corps mou, quasi identique à celui de Timon-David.

L'autre stéréotype du corps de l'irrégulier concerne la sexualité. Elle résulte d'impulsivités incontrôlables du corps, comme le disait Heuyer. Jean, 17 ans, vif, alerte, musclé, a des réactions psychomotrices violentes et désordonnées. Son comportement moteur est perturbé, la coordination de ses gestes ne s'effectue pratiquement pas. Il a une attitude contractée. Il ignore, après l'effort, la phase de retour au calme. Son corps lui échappe donc, il a besoin d'excitation, nous dit l'observateur : "Il recherche l'assouvissement par des contacts douteux avec ses camarades. " (81) La lecture bienveillante du corps reproduit les stéréotypes archaïques du corps du délinquant.

En sera-t-il toujours ainsi ? De Sambucy, médecin scolaire, spécialiste de gymnastique corrective, loue, dans la préface de l'ouvrage de Belligon, les qualités de redoutable scrutateur de l'auteur. "L'œil morphologique » lui permet de classer " les faces anormales, les crânes aplatis transversalement, les poitrines aplaties, les cous mal plantés, les ventres en besace ", signes visibles de la "France déficiente ". ${ }^{(82)}$ Belligon scrute le corps nu, de bas en haut, à distance de profil et de dos, puis de près. Les 15 segments du corps étudiés sont notés de 0 à 5 . L'œil morphologique, aidé des mesures et du graphique, lit rationnellement les corps. Il les désigne par des mots aux sens forts, qui frappent les imaginaires et construisent des stéréotypes. Ces corps désignent des " retardés, des caractériels ou des délinquants ». ${ }^{\left({ }^{83}\right)}$ Ces derniers se voient dans la colonne 5 de ses fiches morphologiques, celle des « déficients morphologiques ", ${ }^{(84)}$ dont le corps est marqué par la faiblesse, avec les ventres mous, les dos tordus, les pieds affaissés, les épaules voûtées.

Le regard de l'éducateur doit scruter l'individu dans sa totalité. Selon Belligon, ${ }^{(85)}$ il enrichit les classifications limitées des tests d'intelligence, ainsi que les concepts spécialisés des neurologues. Au-delà de l'aspect morphologique, l'œil voit « les possibilités fonctionnelles, les impossibilités de certains mouvements, les liaisons psycho et neuro-motrices $»{ }^{(86)}$

Il note que ces enfants, « les idiots, les imbéciles, les débiles (profonds, nets ou légers) ", qui sont différents des autres, généralement en retard scolaire, 
" les sous-normaux », ${ }^{(87)}$ sont maladroits. Le contrôle du corps leur échappe, mais pas au regard du spécialiste qui pense lire tout ce que le sujet ne dit pas et que son corps montre. Cela se constate dans des tics visibles, dans le besoin incontrôlable de mouvements rapides et incomplets, dans l'incoordination de mouvements, dans un complexe d'infériorité ${ }^{(88)}$ et, plus généralement, dans leur « infantilisme moteur ». ${ }^{(89)}$

Ce dernier se voit aussi dans le tonus anormal de ces enfants, trop raides ou trop laxes. L'hypo-tonus postural du laxe résulte d'une " paresse du muscle faible ", ${ }^{(90)}$ qui se constate chez les délinquants, dit-il. C'est pourquoi le travail de rééducation posturale leur est particulièrement conseillé. On constate chez les délinquants, explique Belligon, "qu’à une amélioration statique correspond une amélioration du comportement », qui se verrait, selon lui, à « la belle allure physique et morale $»{ }^{(91)}$

Le résultat obtenu découle de la dépossession d'une part importante d'euxmêmes, dans le but de reconstruire leur corps pour l'adapter aux normes physiques, sociales et morales. ${ }^{(92)}$

C'est la reproduction à l'identique de ce qui se fait au même moment dans le groupe scolaire de Vanves, où on loue les changements d'attitude, l'allure preste et "plus racée ", avec une classe d'enfants en retard scolaire et présentant un tableau morphologique semblable à celui que dépeint Belligon. ${ }^{(93)}$ Le même processus est à l'œuvre dans les centres de rééducation physique et dans les classes de perfectionnement. Physiquement, l'ensemble de la jeunesse en difficulté, des " enfants inadaptés ", ${ }^{(94)}$ est soumis au même traitement. En effet, leur corps est marqué essentiellement par l'anormalité qui se voit dans la faiblesse. Cette catégorie, aux contours flous, est le concept central du regard de l'observateur averti ou plus simplement curieux. Elle est pourtant au cœur du stéréotype corporel du jeune inadapté, qui s'enrichit au fil de l'évolution des connaissances. Ainsi, l'approche biotypologique va prendre en compte les données de la psychomotricité, sans changer le regard sur le corps de cette catégorie de la jeunesse. Il est toujours chétif, atteint de " déformations structurales entraînant des insuffisances respiratoires, circulatoires et musculaires ». ${ }^{(95)}$ Ces enfants montrent une apathie résistante ou une exubérance incontrôlable entraînant des " troubles du comportement ". Le corps de l'inadapté est maladroit « dans les mouvements digitaux, amples, alternatifs,
(87) Ibid.

(88) Ibid.

(89) Ibid., p. 554.

(90) Ibid., p. 555.

(91) Ibid.

(92) La transformation du corps de l'irrégulier est l'aboutissement d'un long processus, dont on peut percevoir quelques étapes dès 1844 . Une des finalités assignées, alors, à la colonie agricole de Bonneval, par le baron de Jessaint, préfet d'Eureet-Loir, est de corriger, grâce au développement des facultés physiques des enfants, « qui auront besoin d'une santé robuste ", les vices de la première éducation « qui ont perverti ces malheureux ». Ces derniers doivent reconstruire leur corps pour le rendre adapté à sa destinée sociale, valet de ferme.

(93) Philippe Encausse, op. cit., p. 9. 
(94) Yves Camus, André Le Maux, "L'éducation physique dans les classes de perfectionnement ", EPS, $\mathrm{n}^{\circ} 79,1966$, p. 19-20.
(95) Ibid.
(96) Ibid.
(97) Ibid.

(98) Louis Tanon, "L'expérience scolaire de Vanves ", Bulletin de l'Académie nationale de médecine, 136, 29-07, 1952, p. 501-506.

Le professeur Tanon, personnalité importante de l'Académie nationale de médecine, dont il sera le président en 1964, et qui siège à la commission hygiène depuis 1935, fait une communication sur l'expérience de Vanves, qu'il appelle « notre expérience scolaire ", et qui, selon lui, mérite l'audience de l'Académie. Sa communication souligne la valeur éducative de la honte du corps dans l'équilibre ". Il est aussi maladroit dans les domaines sensori-moteurs, audio-moteurs, visio-moteurs, idéo-praxiques, et aussi dans le temps, car les mouvements arythmiques sont ou trop lents ou trop rapides. ${ }^{(97)}$ Les bilans psychomoteurs du corps décrivent en fait un " corps physiologiquement anormal ", ${ }^{(98)}$ symbole de déviance dont parle Tanon. Le regard des spécialistes sur ces corps et les mots utilisés pour en parler ont pour fonction essentielle de protéger la société en inscrivant le corps différent dans la marginalité, physiologique, morale et sociale.

Le regard optimiste de Sinoir a été suivi de discours ambigus et souvent contradictoires, encore fortement teintés de références archaïques malgré les références nouvelles à la psychomotricité.

\section{La typologie de Sheldon, le regard total et l'observation physique des délinquants}

Cinq ans après Dijon, un nouveau colloque national de l'UNAR, à Strasbourg, en 1957, dresse le bilan de ce qui se fait en matière d'observation dans l'éducation surveillée. On y apprend que les mensurations et les examens morphologiques ne sont pas pratiqués partout. C'est une erreur, selon le rapporteur, si l'on veut dresser un tableau complet de la personnalité, car il y a, dans cette population, une grande proportion « d'inadaptés dans la sphère physio-somatique ». ${ }^{(99)}$ La rééducation physique ne peut commencer qu’après un examen et des observations, en activité, les plus précises possible. L'activité motrice est minutieusement détaillée. On y observe, en particulier, la résistance à l'effort, l'adresse corporelle et générale, le rythme personnel d'action, le sens de l'équilibre, la sensibilité au vertige, l'adaptabilité aux situations nouvelles. Il est fait référence aux succès de l'expérience de Vanves. On est dans une perspective éducative. Le jeune irrégulier a une existence propre, il est un être singulier doté d'une personnalité. L'observation de la personnalité, au cœur de la nouvelle approche des jeunes irréguliers, désormais rééducables depuis les textes de 1945, doit être rationalisée.

En effet, pour pouvoir fonctionner, cette ordonnance organise un processus qui porte l'attention sur le sujet et non plus sur l'acte. Elle a besoin, pour cela, de disposer d'un savoir complet sur la personnalité du délinquant. Il convient donc de réétudier les corrélations entre les domaines physiques, intellectuels, 
affectifs et sociaux. A priori, on ne peut plus fonctionner à partir de suppositions comme Binet. Par ailleurs, les références scientifiques ont changé. C'est à Claparède, Decroly, Piaget et Wallon que se réfere Durand. Les approches très normatives de Paul-Boncour et de Heuyer sont délaissées, car on ne parle plus d'enfance coupable. Les références aux tares et aux dégénérescences ne sont plus de mise. La tâche est complexe, car il convient de trouver une nouvelle approche, tentant de lier des éléments propres à rendre compte de la personnalité en actes pendant les séances d'éducation physique. C'est le comportement qu'il faut étudier, et c'est précisément ce qu'ont toujours voulu saisir les observations antérieures, comme celle de Timon-David, par exemple.

Comment ne pas redire la même chose ? Quels signes corporels observer et comment les interpréter ? Lorsqu'on regarde les fiches d'observation construites dans les années 1950, on est frappé de constater combien le corps peut éveiller le regard. C'est évident avec Belligon. Cela l'est encore plus sur le terrain des centres d'éducation surveillée. Il n'est pas rare de rencontrer des fiches contenant plus de 180 items censés rendre compte des comportements des jeunes irréguliers.

Une commission a été mise en place par le garde des Sceaux, au centre de Vaucresson, ${ }^{(100)}$ pendant les années 1955 et 1956, pour harmoniser l'observation par l'éducation physique et les sports. Il s'agit de dresser un bilan de la personnalité du mineur. Cette commission est composée de sept membres. La personnalité marquante en est le docteur Bize, ${ }^{(101)}$ qui est professeur aux Arts et Métiers et conseiller médical de la direction de l'Éducation surveillée. Il est l'auteur de plusieurs ouvrages sur la bio-typologie. Il collaborera pendant 25 ans avec la direction de l'Education surveillée où son approche de la connaissance scientifique des mineurs, à partir de la morphologie, le place en décalage avec d'autres approches. Il rédige, en 1953, un texte sur les prédispositions à l'état dangereux. Il se situe dans une perspective éclectique en raison de la diversité des déterminants de la " criminogénèse ". ${ }^{(102)}$ Il invite à prendre quelques distances avec la part de l'irréductibilité des facteurs relevant de la biologie, en prenant une perspective dynamique. Enfin, il reconnaît le rôle des structures sociales qui peuvent être des facteurs de désadaptation. Néanmoins, son travail consiste à construire, le plus justement possible, le profil du criminel observé, afin que la peine qui lui sera appliquée tienne qu'éprouvent les enfants à

la fin de l'expérience :

« Ils ont honte de paraittre sale à la piscine. "

(99) Georges Durand,

«Le point de vue des

moniteurs d'éducation

physique ", Sauvegarde de

l'enfance, $\mathrm{n}^{\circ} 1 / 2 / 3$,

UNAR, Paris, 1958,

p. 323. L'auteur est

professeur d'éducation

physique au centre de

formation de l'Éducation

surveillée de Vaucresson.

(100) Georges Durand

fait partie de la com-

mission mise en place

à Vaucresson.

(101) Pour une étude complète sur Bize, on se reportera à l'article de Jean-Pierre Jurmand, «Bize : un médecin à l'Éducation surveillée, ou les sciences de l'observation entre archaïsme et modernité ", le Temps de l'histoire, $\mathrm{n}^{\circ} 6$, octobre 2004. 
(102) Paul-René Bize,

«Le problème de la structure somato-psychique prédisposant à l'état dangereux ", Deuxième cours international de criminologie, Paris, 1953 , p. 33.

(103) Ibid., p. 8.

(104) Ibid.

(105) Ibid., p. 9.

(106) Ibid., p. 8.

(107) William Herbert Sheldon, Les variétés de la constitution physique de l'homme, Paris, PUF, 1950.

Reprenant ces travaux,

Malard, « La morphologie détermine-t-elle le caractère et le comportement ? ", Psychologie, n 24, 1972 , valide la classification de Sheldon et accrédite la thèse de la validité du facteur morphologique, en affirmant une prédominance de mésomorphes chez les délinquants étudiés. L'auteur souligne toutefois, avec prudence en conclusion, que compte de ses caractéristiques propres, analysées à travers les facteurs prédisposant à la délinquance. Cette approche vient contredire la prise de distance vis-à-vis de l'irréductibilité des facteurs.

Toutefois, Bize récuse le terme de dégénérescence appliqué aux mineurs difficiles, sauf pour une minorité « très primitive morphologiquement ». ${ }^{(103)} \mathrm{Il}$ parle " de traits inesthétiques ", ${ }^{(104)}$ parfois rejetés par l'environnement social. Il existe aussi " les faces d'ange ", des précoces sexuels, des robustes, qui prennent « le chemin de l'imposture ». ${ }^{(105)}$ Les facteurs de délinquance sont composés de facteurs négatifs et positifs.

La personnalité constitue un tout, prenant en compte l'ensemble des facteurs. Cependant, la recherche d'unité et de singularité du sujet, perceptible dans une typologie corporelle, vient écraser la diversité des facteurs. L'idée du codage diversifié du corps est contredite par l'utilisation des types corporels.

De l'étude de 25 dossiers, Bize déduit les données cliniques suivantes : « La masse des guetteurs est formée de chétifs passifs, les hommes de choc se recrutent parmi les athlétiques. " ${ }^{(106)}$ Les délinquants sont majoritairement de type corporel athlétoïde (bréviligne asthénique), classés selon Sheldon parmi les mésomorphes. ${ }^{(107)}$ Après avoir étudié les corps de 4.000 étudiants de Harvard, Sheldon a dégagé trois grands types corporels, qu'il classe sur une échelle de 1 à 7 , ce qui lui donne des possibilités immenses de combinaison. Ses trois composantes de base sont : l'endomorphisme, avec prédominance des rondeurs, le mésomorphisme, au physique anormalement lourd, rude et rectangulaire, et l'ectomorphisme, au physique linéaire et fragile. Il étudie l'ensemble du corps y compris la pilosité. Il pense avoir trouvé les causes physiques de la délinquance (p. 289-293), faiblesse et mollesse intérieure, des traits nettement passifs, dépendants et même féminins, avec une rudesse manifeste.

La catégorie des mésomorphes prédominerait parmi les délinquants. On la retrouverait dans 60,1\% des cas selon Bize. D'autres caractéristiques distinguent les délinquants. Leur tonus général révèle une inattention au corps : «Les délinquants sont sales, [...] grossiers dans leur tenue et veules dans leur attitude. "(108) Si l'on rencontre des passifs apathiques et des actifs combatifs, la plupart des délinquants sont des hédonistes ayant horreur de l'effort. Leur conduite est marquée par "l'entrainabilité " ${ }^{(109)}$ et une capacité de contrôle limitée. L'impulsivité est réduite dans « l'état délinquantiel » ${ }^{(110)}$ à un court-circuit, dans 
lequel le feed-back négatif, celui de l'éthique, selon Bize, ne joue aucun rôle. Enfin, on note une absence " du facteur d'intimidabilité » et donc de peur, ainsi que celle du facteur de "l'émotion tendre ». ${ }^{(11)}$ La pensée de Bize, étayée par des observations cliniques, par une lecture d'écrits scientifiques sur le sujet, est écrasée par la recherche de types singuliers et des facteurs prédominants. Par ailleurs, les concepts utilisés rendent compte d'un processus non modifiable.

L'utilisation de formules fortes, telles que « l'arriération affective d'évolution ", ${ }^{(112)}$ laissent penser que quelques facteurs pourraient suffire à comprendre la personnalité du jeune. Enfin, la notion d'état délinquantiel bloque toute approche de la personnalité essayant de comprendre l'ensemble des facteurs ayant conduit ce jeune à entrer dans l'univers irrégulier. Un phénomène complexe, comme celui de la délinquance, devient un état de fait, pour lequel l'observation multi-dimensionnelle de la personnalité fournit des réponses. Or, la plupart des concepts utilisés par Bize font partie des stéréotypes du délinquant, lentement construits, travaillés par le temps et l'histoire, ayant force d'évidence.

L'ensemble du processus analysé se retrouve dans le travail de la commission travaillant à Vaucresson. L'examen du corps, dit-on, doit classer les individus dans les groupes d'éducation physique. Après les mesures morpho-physiologiques habituelles, il faut classer les sujets en 5 groupes, de l'aptitude totale à l'inaptitude totale. Il faut rechercher, notamment, l'état réel de la robusticité ou du chétivisme, la gracilité musculaire, la faiblesse des parois, le névrosisme. ${ }^{(113)}$ On cherchera les signes d'énergie ou d'asthénie. ${ }^{(114)}$ Dans l'exécution des gestes sportifs et gymniques, on observera notamment les qualités gestuelles. On notera l'absence de mouvements anormaux, les tremblements, toute " anomalie tempéramentale exposant à des ratés de l'automatisme ». ${ }^{(15)}$

L'observateur dispose d'un système de codage du corps qui fournit l'élément à noter et son interprétation. Ensuite, il convient d'apprécier « la composante carrure ". ${ }^{(116)}$

On se référera, selon Bize, à la typologie de Sigaud ${ }^{(117)}$ dont il retient la simplicité de ses 4 types humains : respiratoire, gastro-intestinal, musculaire, cérébro-spinal ou à celle de Sheldon. On peut ainsi, d'après Bize, s'appuyer sur un corpus de 5 types humains, en fonction de la forme du buste, du type de faciès, des contours (adipeux, musculeux, estompés), des chevilles, de l'ossature et de la composition pigmentaire (claire, congestive, colorée, terreuse).

\author{
" le problème du rôle de la \\ constitution physique \\ dans les conduites \\ humaines demeure". \\ Ce qui, en définitive \\ contredit toute la thèse \\ de l'article.
}

(108) Paul-René Bize, op. cit., p. 14.

(109) Ibid., p. 15.

(110) Ibid., p. 30.

(111) Ibid.

(112) Ibid., p. 28.

(113) L'observation par l'éducation physique et les sports, Vaucresson, 1957 , p. 19.

(114) Ibid., p. 25.

(115) Ibid., p. 28.

(116) Ibid., p. 37-38.

(117) Claude Sigaud est un gastro-entérologue lyonnais, auteur en 1914 d'un ouvrage sur la forme humaine et sa signification. La Société d'étude des formes humaines, créée en 1923 pour étudier l'hérédité, 


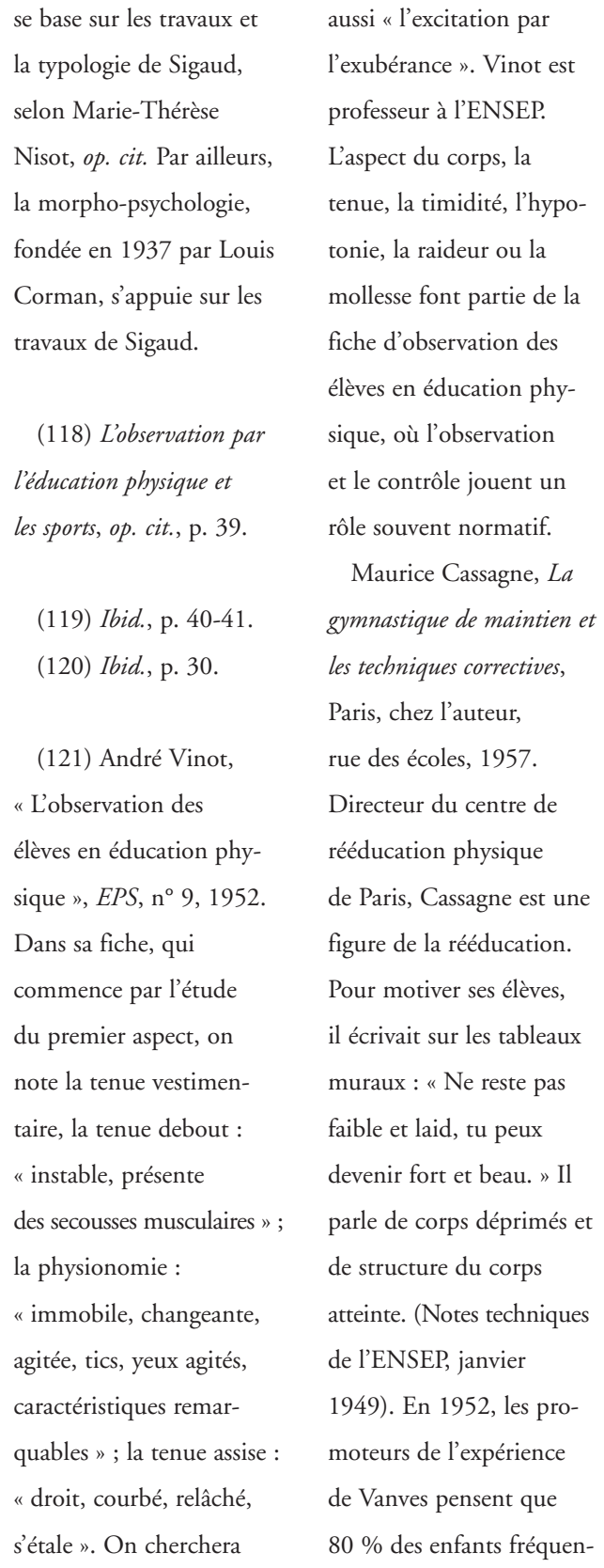

La multiplicité des facteurs, l'imprécision qui les caractérise, limitent fortement la dimension objective de l'observation. Interpréter un raté dans l'exécution d'un geste comme une anomalie "tempéramentale " est tout à fait abusif. On est ici au cœur du stéréotype corporel. On y reste avec l'étude de la composante tonique qui cherche à déceler l'état de tension des muscles. Est-il permanent, sans repos, ou bien mou ou "dépressible " ? (118) Vient ensuite la composante sexuelle, masculine et féminine, distribuée en cinq catégories. Elles vont du type hyper viril ou hyper féminin, au type infantile. Les types sexuels sont redondants avec les types corporels en ce qui concerne les traits, l'ossature, le bassin. Par contre, la peau est ici étudiée selon sa texture. Est-elle rude et acnéique, signe de virilité, fine sans acné, glabre, signe de féminité ?

Autre signe corporel à noter, la pilosité pubienne. Elle est en losange chez les garçons et en triangle chez les filles. On recherchera une inversion chez le type masculin gynoïde ou intersexuel, avec sa pilosité en triangle, et chez le type féminin viriloïde ou intersexuel, avec sa pilosité en losange. ${ }^{(119)}$ Dans cette perspective, l'inversion, le signe corporel hors norme, est le facteur posant problème. Or, nous sommes ici en présence de caractères corporels biologiques, indépendants de la volonté du sujet. C'est le retour de l'hérédité et aussi des références archaïques dans l'observation du corps. On parle encore de sujet naturellement sale, qui se complait dans la malpropreté. Cette dernière va au-delà de l'usage de soins hygiéniques. C'est de la pureté intérieure dont il est question, celle du sujet sain, propre, doué d'intelligence physique, qui se distingue par « une sorte d'aisance naturelle, quasi intuitive, à trouver d'emblée la solution la meilleure ", ${ }^{(120)}$ celle du droit chemin. 
Le regard scrute l'intime du corps et il cherche à découvrir par ce biais le monde intérieur, le sujet profond. Une approche similaire aura court dans l'éducation physique scolaire, ${ }^{(121)}$ où les corps faibles, tordus, sont souvent compris comme des signes de déviance.

De Binet au début du XXème siècle à Belligon et Bize dans les années 1950, le regard sur le corps devient de plus en plus précis. Aidé de fiches, nourri de multiples observations, il devient total et pense lire la vérité du sujet. Le regard s'approprie l'ensemble du corps, marqué par l'anormalité et l'obligation de changer.

On voit se construire, pendant cinquante ans, au nom de nouvelles références, un modèle du corps toujours normatif, fortement stéréotypé, archaïque. La France déficiente de Belligon et Bize est-elle si différente de celle que dénonçaient Bureau, Bourgeois et Buisson dans les années 1920 ?

La tentative de renouvellement du regard sur le corps des délinquants, dans la période qui suit la deuxième guerre mondiale, donnera peu de résultat. Il est toujours violent, car il tend à construire chez le jeune une distance vis-à-vis de lui-même. Les hygiénistes du début du XXème siècle ont joué un rôle décisif dans le processus de dépossession du corps au profit des valeurs morales. ${ }^{(122)}$

La lecture du corps est prise dans des déterminations culturelles et symboliques fortes. Le corps est aussi engagé dans la scène sociale. Là, le corps du délinquant, de l'instable scolaire, vient produire du désordre et de la peur. Le regard est désormais total, rien ne lui échappe. Il est violent et le corps observé lui est entièrement soumis. Ce processus, qui s'applique prioritairement aux jeunes irréguliers, est aussi valable pour tous les autres. Le résultat en est un corps déshumanisé. tant les écoles primaires (tout au moins à Paris) sont atteints d'imperfection physique. C'est la reproduction du discours sur la race en danger.

(122) «Si l’on professe que je suis tout au moins le propriétaire de mon corps, dit Bureau, comment faire accepter les valeurs supérieures du sentiment de sacrifice et d'abnégation ? ", Pour la vie. Revue de repopulation et d'hygiène morale et sociale, $\mathrm{n}^{\circ} 20,1916$. 